\title{
Percutaneous closure of secundum atrial septal defect with a new self centring device ("angel wings")
}

C Rickers, C Hamm, H Stern, T Hofmann, O Franzen, R Schräder, H Sievert, D Schranz, I Michel-Behnke, J Vogt, D Kececioglu, W Sebening, A Eicken, H Meyer, W Matthies, F Kleber, J Hug, J Weil

Department of Paediatric Cardiology, University Hospital Eppendorf, Hamburg, Germany

C Rickers

H Stern

J Weil

Department of Cardiology, University Hospital Eppendorf

C Hamm

T Hofmann

O Franzen

Department of Cardiology, Hospital Bethanien, Frankfurt, Germany R Schräder H Sievert

Department of Paediatric Cardiology, University Hospital Gießen, Germany

D Schranz

I Michel-Benke

Department of Paediatric Cardiology, University Hospital,

Münster, Germany

J Vogt

D Kececioglu

Department of Paediatric Cardiology, Heart Center Munich, Germany

W Sebening

A Eicken

Department of Cardiology, Heart Center Bad

Oeynhausen, Germany W Matthies

H Meyer

Department of Cardiology, Charité Campus Virchow Klinikum, Humboldt University of Berlin and German Heart Institute Berlin, Germany

F Kleber

J Hug

Correspondence to: Dr C Rickers, University Hospital Eppendorf, Department of Paediatric Cardiology, Martinistrasse 5220246 Hamburg, Germany.

Accepted for publication 24 June 1998
Abstract

Objective-To investigate the safety, efficacy, and clinical application of a new self centring device ("angel wings") for closure of secundum atrial septal defects (ASD II) and persistent foramen ovale in all age groups.

Design-Multicentre, prospective, nonrandomised study.

Patients-Inclusion criteria: defects with an occlusive diameter of $\leqslant 20 \mathrm{~mm}$ and a surrounding rim of $>4 \mathrm{~mm}$; body weight $>10 \mathrm{~kg}$; and an indication for surgical closure of secundum atrial septal defect. Additionally, there were compassionate indications for closure in patients with persistent foramen ovale.

Interventions-Defects were closed by a transcatheter device consisting of two square frames made of superelastic nitinol wire. The frames are covered by elastic polyester fabric, which is sewn together at a central circle. All procedures, except for three interventions that were carried out under sedation, were performed under general anaesthesia using transoesophageal echocardiography and fluoroscopy to monitor intervention.

Results-Closure was attempted in 75 (71\%) of 105 patients. An ASD II was present in 35 children and 15 adults. A persistent foramen ovale was present in 25 adults with suspected paradoxical embolism. Transcatheter closure was unsuccessful in three children and crossover to surgery was required. Residual shunts were found in 20 patients $(27 \%)$ immediately after the procedure. A transient atrioventricular third degree block occurred in three patients $(4 \%)$ and the right atrial disk was not fully deployed in three. A minor shunt $(<3 \mathrm{~mm})$ was present in only three $(4 \%)$ of 72 patients during follow up of 1-17 months. Blood clots on the right atrial disks in two patients (one required lysis) were seen during follow up transoesophageal echocardiography. Serious complications demanding surgical removal of the device occurred in three patients. One patient had haemopericardial tamponade because of an aortic lesion. Left atrial thrombus formation due to an unfolded right atrial disk was found in a second patient and dislodgement of the left atrial disk resulted in a large residual shunt in a third.
Conclusions-Percutaneous closure of a central ASD with a diameter $\leqslant 20 \mathrm{~mm}$ in paediatric and adult patients is feasible and effective with this new device. It is a promising alternative to surgical closure. Modifications of the design, however, seem to be mandatory as $4 \%$ of patients developed serious complications. (Heart 1998;80:517-521)

Keywords: secundum atrial septal defect; persistent foramen ovale; interventional cardiology; transcatheter occlusion

Occlusion of a secundum atrial septal defect (ASD) using a transcatheter double disk device was first reported by King and Mills in $1976 .{ }^{1}$ The large catheter size (23 F) limited its applicability in small children. In 1983 Rashkind $^{2}{ }^{3}$ described a single umbrella device $(15 \mathrm{~F})$ that relied on "fish hooks" to ensure attachment to the left side of the atrial septum and was therefore often poorly centred. In the following two decades other double disk devices ${ }^{4-6}$ were investigated for transvenous closure of ASD or patent foramen ovale. ${ }^{7}$ None of these devices was approved for routine clinical use because of several drawbacks such as persisting residual leakage,${ }^{58}$ high failure rate, ${ }^{7}$ wire fracture, ${ }^{9} 10$ and embolisation of the device. ${ }^{11}$ A novel self centring double disk device ("angel wings") was first introduced by Das et al. ${ }^{12}{ }^{13}$ This device consists of two square frames made of superelastic nitinol wire (fig 1). Each square frame has eight eyelets, four corner eyelets, and four at the midpoint of each leg, functioning as torsion springs. The wire frames are covered with polyester tissue which is sewn together at a central circle, the diameter of which is approximately half the size of the disk. The device is loaded in a 12 or $13 \mathrm{~F}$ delivery catheter (fig 2) and is available in a range of sizes from $12 \mathrm{~mm}$ to $35 \mathrm{~mm}$. The technique of transcatheter closure of ASD with the angel wings device has been described in detail. ${ }^{12}{ }^{13}$ We now report the first clinical results and mid-term follow up data with this new self centring device in Germany.

Patients and methods

Patients with an interatrial communication were considered for percutaneous closure with the angel wings device if the procedure was clinically indicated because of a significant left to right shunt or after suspected paradoxical 


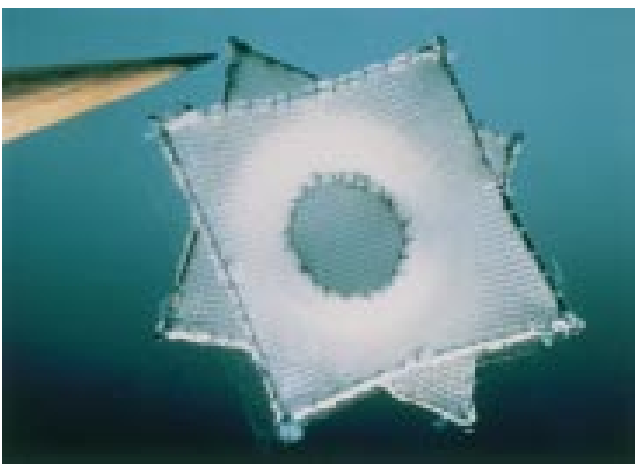

Figure 1 Angel wings device consisting of two square frames made of superelastic nitinol wire. The wire frames are covered with polyester tissue sewn together at a central circle, the diameter of which is approximately half the size of the disk.

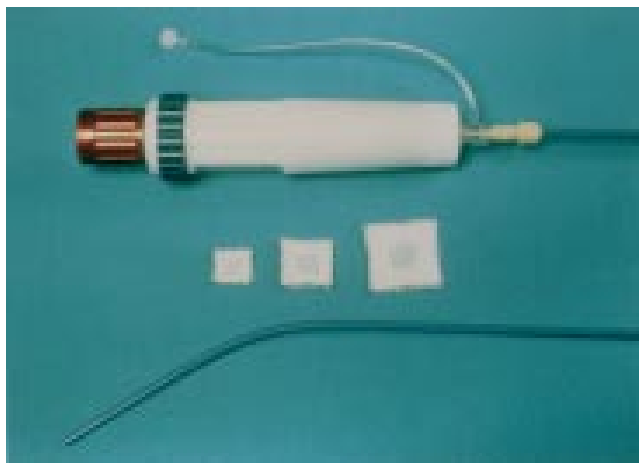

Figure 2 The standard device is available in a range of sizes from $18 \mathrm{~mm}$ to $35 \mathrm{~mm}$. It has a delivery system that consists of a control handle connected to an 11,12, or $13 \mathrm{~F}$ gauge delivery catheter.

embolism. A total of 105 patients were studied: both paediatric $(n=66)$ and adult $(n=39)$. Patients fulfilled the following inclusion criteria. (1) Central defects within the fossa ovalis (secundum ASDs and patent foramen ovale) were less than $20 \mathrm{~mm}$ (occlusive diameter) at a distance of at least $4 \mathrm{~mm}$ from the coronary sinus, atrioventricular valves, and pulmonary veins. (2) Dilatation of the right ventricle and paradoxical motion of interventricular septum were evidence for right ventricular volume overload as seen on echocardiography. (3) The ratio of pulmonary blood flow to systemic blood flow (Qp:Qs) was 1.5:1 or greater. (4) A left heart or carotid artery source was not present in patients with suspected paradoxical embolism (embolic stroke, transient focal neurological deficit, or peripheral arterial embolism), and each had an interatrial communication with intermittent or continuous right to left shunting as seen on contrast echocardiography. Complete neurological evaluation was normal, and migraine and seizures were excluded. (5) Patients with significant residual shunts after a fenestrated Fontan operation.

Exclusion criteria were: interatrial communication other than fossa ovalis defect; abnormal pulmonary venous drainage; associated congenital abnormality requiring cardiac surgery; severe pulmonary arterial hypertension with bidirectional or right to left shunting; the presence of sepsis or malignancy in patients in whom life expectancy was less than three years; intracardiac thrombi as seen on echocardiography; history of severe allergy to iodinated contrast agents; and body weight of less than $10 \mathrm{~kg}$.

Patients fulfilling the inclusion criteria were offered the option of transvenous angel wings closure or surgical closure. Devices were implanted according to research protocols approved by the American Food and Drug Administration. Informed consent was obtained using forms approved by the ethics committees of the involved centres.

\section{CARDIAC CATHETERISATION AND CLOSURE} TECHNIQUE

Routine diagnostic cardiac catheterisation was used to determine the haemodynamic significance of an ASD. Systemic pressure was monitored from a femoral arterial access. General anaesthesia and continuous transoesophageal echocardiography (TOE) supervision for optimal results were performed in almost all patients. Three procedures were performed under sedation. "Balloon sizing" with contrast filled balloons (diluted with saline 1:4) was used to test occlusion of atrial defects. ${ }^{14}{ }^{15}$ The balloon volume was gradually decreased to a minimum diameter that could not be pulled across the defect from left to right and vice versa. This diameter represents the balloon

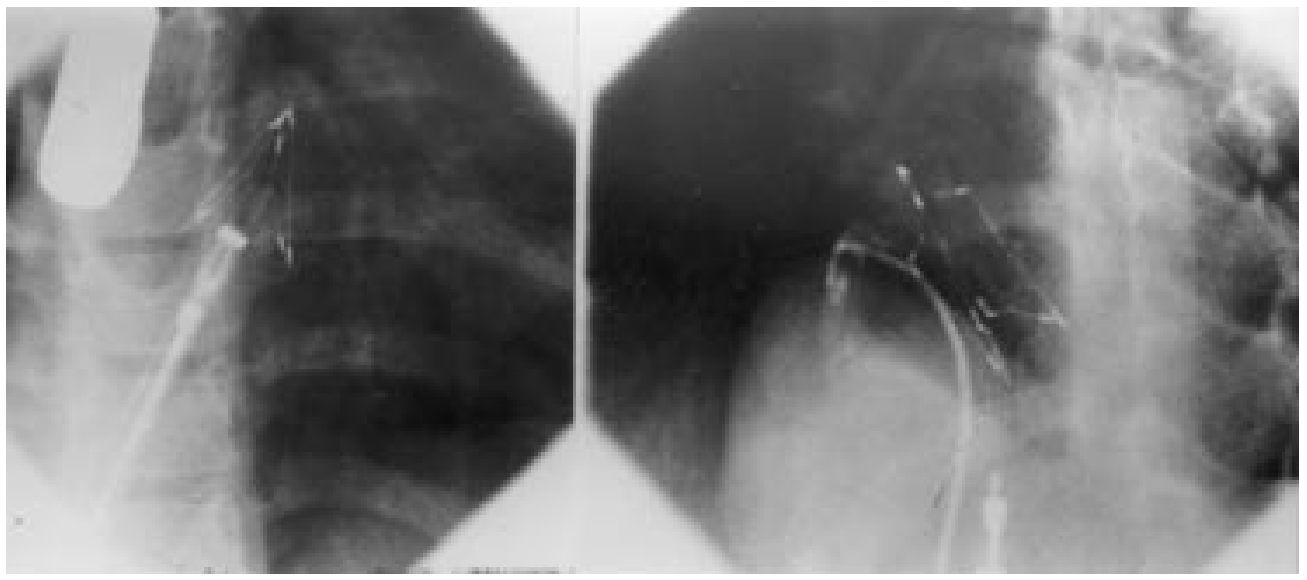

Figure 3 Delivery of the angel wings device. (Left) Typical arrow-like appearance of the left atrial disk before full deployment (right anterior oblique view). (Right) The left and right atrial disks are delivered on the two sides of the atrial septum and the device is released. 


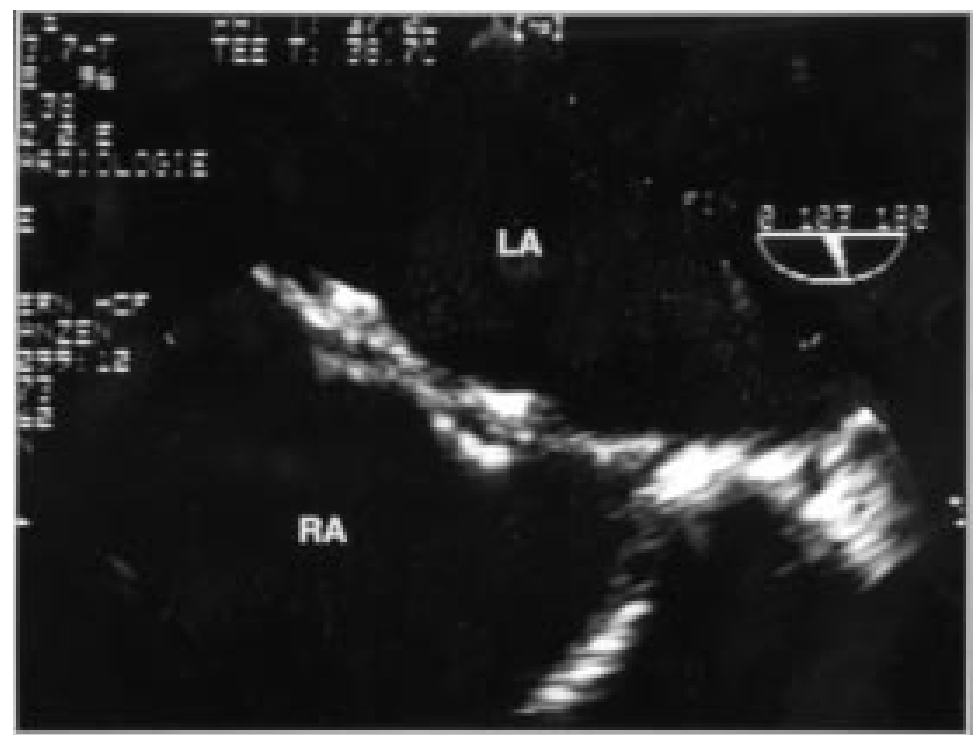

Figure 4 Transoesophageal echocardiogram showing ideal positioning of the device, which has a typical sandwich-like appearance. $L A$, left atrium; $R A$, right atrium.

Table 1 Patient characteristics and implantation data

\begin{tabular}{lllllll}
\hline Defect & $n$ & $\begin{array}{l}\text { Age } \\
\text { (years) }\end{array}$ & $\begin{array}{l}\text { Weight } \\
(\mathrm{kg})\end{array}$ & Qp:Qs & $\begin{array}{l}\text { Occlusive } \\
\text { diameter (mm) }\end{array}$ & $\begin{array}{l}\text { Fluoroscopy } \\
\text { time (min) }\end{array}$ \\
\hline ASD II (paediatric) & 35 & $7.8(4)$ & $26(12)$ & $1.5-2.9: 1$ & $13(3)$ & $21.6(12)$ \\
ASD II (adults) & 15 & $50(11)$ & $79(16)$ & $1.5-2.7: 1$ & $16(4)$ & $22.8(9)$ \\
PFO & 25 & $44(13)$ & $75(12)$ & - & $8(4)$ & $17.3(5)$ \\
\hline
\end{tabular}

ASD, atrial septal defect; PFO, persistent foramen ovale

occlusive diameter. The appropriate size of the device was determined by multiplying the occlusive diameter by 1.5 . Thereafter a suitable sized Mullin's sheath (Cook, New Jersey, USA) was positioned in the mid-left atrium. Mullin's sheath was continuously flushed with isotonic saline to prevent air embolism. The left atrial disk was deployed by clockwise torquing of the blue actuator ring (fig 2). The right atrial disk was deployed after withdrawal of the fully opened left atrial disk against the interatrial septum. The correct position of the device was ensured by TOE (fig 4) and fluoroscopy. The device was released by torquing the red release knob of the control handle in a counterclockwise direction and pulling back.

PATIENT MANAGEMENT AND FOLLOW UP

Patients were fully heparinised (100 units $/ \mathrm{kg}$ ) to achieve an activated clotting time of about 300 seconds at the beginning of the procedure: each received antibiotic prophylaxis. Patients were discharged one to three days after closure.
Acetylsalicylic acid (2-3 mg/kg/day) was prescribed for six months. Colour flow Doppler echocardiography, standard ECG, and chest radiography were performed before discharge and three months after clinical investigation. TOE was undertaken between three and six months after the procedure.

\section{Results}

A total of 105 patients were considered for transcatheter closure of ASD or persistent foramen ovale. Closure was not attempted in 30 patients: multiple lesions were present in six and the occlusive diameter of the balloon was either more than $20 \mathrm{~mm}$ or too large in relation to the atrial septum in 24 . Device closure was attempted in the remaining 75 patients $(71 \%)$. An ASD II was present in 35 children (mean (SD) age 7.8 (4.0) years (range 4.3-16), mean (SD) weight 26 (12) kg (range 13-48), mean (SD) balloon occlusive diameter 13 (3) $\mathrm{mm}$ (range 10-20)) and 15 adults (age 50 (11) years (range 17-72), weight 79 (16) kg (range 56-110), balloon occlusive diameter 16 (4) $\mathrm{mm}$ (range 15-20)) (table 1). The Qp:Qs ratio ranged from $1.5: 1$ to $2.9: 1$ in the children and from $1.5: 1$ to $2.7: 1$ in the adults. Anomalous drainage of pulmonary veins and pulmonary hypertension were not present. A patent foramen ovale was present in 25 adults (age 44 (13) years, weight 75 (12) $\mathrm{kg}$ ) with suspected paradoxical embolism.

Successful transcatheter occlusion of the defect (fig 3) was performed in 72 patients (96\%) using devices with a diameter of between $18 \mathrm{~mm}$ and $30 \mathrm{~mm}$. The closure rate for persistent foramen ovale was $100 \%$. The preferred device size was $22 \mathrm{~mm}$ for paediatric patients; $30 \mathrm{~mm}$ devices were used mostly for adults; $18 \mathrm{~mm}$ and $22 \mathrm{~mm}$ sizes were chosen for closure of persistent foramen ovale (fig 5). Transcatheter closure was unsuccessful in three children (4\%): the left atrial disk pulled into the right atrium in two and the left atrial disk straddled a multifenestrated ASD in the third. As repositioning was unsuccessful and retrieval was not attempted, the device was surgically removed and the ASD closed. All patients recovered from operation without complications. Residual shunts were found in 20 patients $(27 \%)$ immediately after the procedure. A transient atrioventricular third degree block not requiring temporary pacing occurred in three patients $(4 \%)$ and full deployment of the right atrial disk failed but without sequelae

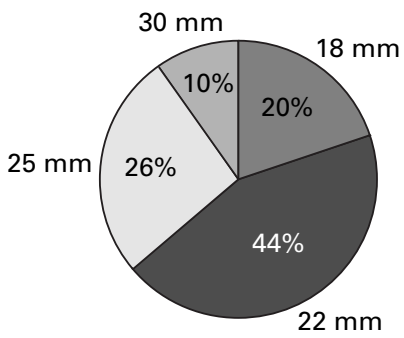

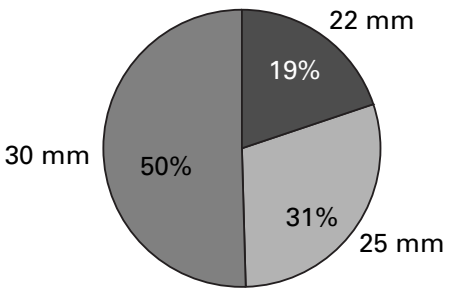

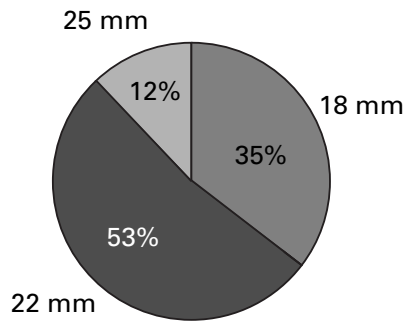

Figure 5 Distribution of angel wing device sizes used in Germany. (A) Atrial septal defect II, paediatric patient; (B) Atrial septal defect II, adult patient; (C) Patent foramen ovale, adult patient. 
was found in three (4\%). The fluoroscopy time was 22.5 (7.3) minutes (range 10.2-42.4 minutes).

A minor shunt $(<3 \mathrm{~mm})$ was still present in three of 72 patients during follow up (1-17 months). Blood clots on the atrial disks in two adult patients after closure of a patent foramen ovale were seen during follow up TOE two and six weeks after device placement. Coumarin was given to the first patient with a thrombus on the right atrial disk. Systemic lysis was started in the second patient who had thrombi on the right and left atrial disks. Cerebral nuclear magnetic imaging and lung scintigraphy showed no abnormality. Coumarin was started for six months after successful lysis. The device had to be surgically removed from three patients. One patient had haemopericardial tamponade four months after closure of ASD. The anterosuperior corner of the device had perforated the aortic root. The ASD and aortic lesion were surgically repaired. A thrombus in the left atrium and right inferior pulmonary vein was seen during routine follow up TOE in a second patient in whom the right atrial disk had failed to completely open. This clot was detected six months postprocedure. Routine TOE showed a displaced left atrial disk and a large residual shunt nine months after closure of an ASD in a third patient. All patients recovered from operation and suffered no other adverse sequelae.

\section{Discussion}

In this initial German series atrial septal defects and persistent foramen ovale were closed in 72 $(96 \%)$ of 75 patients with the new self centring angel wings occluder. The rate of complete closure assessed by TOE was $92 \%$, and residual minor shunting was present in only three patients $(4 \%)$ at follow up. Retrieval of the device was not attempted in the three patients with failed procedures and each underwent surgical repair. Percutaneous retrieval of the device is clearly difficult and a limiting factor. Retrieval in animal models was reported by Das et al,,$^{12}$ but it was not attempted because of the potential risk of perforation.

Major adverse events requiring surgical removal of the device were observed in three patients (4\%). A 16 year old girl (ASD II, $52 \mathrm{~kg}$ ) presented with haemopericardial tamponade four months after device implantation. One of the edges of the device had perforated the aorta. The device $(30 \mathrm{~mm})$ was thought to be too large in relation to the transverse diameter of the atrium, thus giving rise to atrial perforation. A thrombus in the left atrium and the right inferior pulmonary vein was seen during TOE six months postprocedure in a second patient in whom the right atrial disk was not fully deployed. Intraoperatively, the left atrial disk showed a slight deformity that favoured thrombus formation. The unusual form of the left atrial disk was thought to be caused by the right atrial disk, which failed to completely open. Displacement of the left atrial disk in a third patient, an eight year old girl (ASD II, $30 \mathrm{~kg}$ ), resulted in a large residual shunt nine months after closure of an ASD. The device
(22 mm) was removed for fear of perforation. Intraoperative examination showed that the upper left posterior corner was deflected and not attached to the septum.

Injury from the stiff wire framework is the primary concern with this device, although similar complications in more than 270 patients in whom the device was implanted have not been reported. Design modifications-for example, converting the eyelets to the inside of the device, however, are under consideration. It seems mandatory that the selected device has a smaller diagonal diameter than the interatrial septum, to avoid injury of adjacent structures. Thus, careful TOE imaging of the implanted device is required during the procedure. ${ }^{816}$

The angel wings device was designed to avoid many of the limitations of previous devices, such as persistent leakage due to poor centring and a high failure rate because of complex multistep placement. Four devices are undergoing evaluation: the "clamshell" device, the "buttoned" device, the Amplatzer septal occluder, and the atrial septal defect occluder system device. Some have potential disadvantages and certain risks. Limitations of the clamshell device ${ }^{517}$ include persistent residual shunts in as many as $26 \%$ of patients and wire fractures in up to $33 \%$. Recapturing and repositioning are not possible when the umbrellas have unfolded. Carminati et al reported preliminary experience in five patients with a redesigned clamshell device (CardioSEAL). ${ }^{18}$ Drawbacks of the buttoned device ${ }^{6719}$ are a high failure rate $(21 \%$ ) ("unbuttoning" $7 \%$ ), device embolisation, difficult repositioning, and residual shunts (39\%). The atrial septal defect occluder system (ASDOS) device enables positioning of the device within the defect using a guidewire loop which requires simultaneous arterial and venous puncture. The right and left atrial disks are screwed together at a central hub. Transient atrioventricular conduction abnormalities during the procedure have been described. ${ }^{20}$ Procedural success rates for closure of ASD II is between $86 \%$ in adults ${ }^{21}$ and $90 \%$ in paediatric patients. ${ }^{20}$ Residual shunts were seen in $42 \%$ of patients at six months' follow up. The diameter of these three devices must be between 2 and 2.5-fold larger than the diameter of the septal defect, to avoid residual shunting. The angel wings device requires a diameter only 1.5 times larger than the defect because of its self centring mechanism. Sharafuddin and colleagues ${ }^{16} 22$ described the first experimental closure of ASD in dogs also with a new self centring device (Amplatzer septal occluder) made with braided nitinol wire. Chan et al reported promising preliminary experience with this device in 22 children. ${ }^{23}$

The gold standard for closure of ASD is open heart surgery with mortality close to zero. $^{24-28}$ The incidence of significant complications, ${ }^{29}$ such as pneumothorax, postoperative bleeding, pleural effusion requiring chest tube drainage, pericardial effusion, infection, and atrial arrhythmias, varies from $2.5 \%{ }^{29}$ to $13 \% .^{24}$ Residual shunts at follow up are rare, but their incidence can be as high as $7.8 \%$ to 
$16.7 \% .^{26} 3031$ Mild complications (atelectasis, gastrointestinal, urinary tract infection, pleural effusion) have been reported ${ }^{29}$ in $72.9 \%$ of paediatric patients and in $67.5 \%$ of adults. Gala et al reported the results of perioperative complications following surgical closure of ASD II in 232 patients. ${ }^{29}$ They showed that an isolated secundum ASD can be closed safely with minimal mortality but a certain rate of complications has to be accepted, predominantly in adults.

It can be concluded from our mid-term follow up data that this new device seems to be a promising transcatheter technique for occlusion of secundum ASDs in children and adults. Closure of persistent foramen ovale is possible with an excellent success rate $(100 \%)$. No residual shunting was seen but a major adverse event occurred in one patient $(4 \%)$. Blood clots on the atrial disks were seen in two patients $(8 \%)$. Patients with a history of paradoxical embolisation may be more prone to clotting in the absence of a demonstrable clotting abnormality. Coumarin may be indicated until endothelialisaton occurs.

This first device with a self centring mechanism leads to complete occlusion of a central atrial defect in almost all patients. Modification of the design, however, is mandatory, as $4 \%$ of patients developed serious complications. Additionally, a retrieval mechanism needs to be developed. Further randomised clinical trials comparing device closure with surgical closure, as well as long term data, are required.

The first implantations in Germany were supervised by A $M$ Mendelsohn, Childrens Hospital, Cincinnati, Ohio, M P O' Laughlin, Duke University Medical Center, Durham, North Carolina, and A P Salmon, General Hospital, Southampton, $\mathrm{UK}$

\section{Appendix}

Number of patients who underwent device closure

University Hospital Eppendorf, Hamburg

Hospital Bethanien, Frankfurt

University Hospital Gießen

University Hospital Münster

Heart Center Munich

Heart Center Bad Oeynhausen

Virchow Hospital Berlin

1 King TD, Mills NL. Secundum atrial septal defects: non operative closure during cardiac catheterisation. $7 A M A$ 1976;235:2506-9.

2 Rashkind WJ. Transcatheter treatment of congenital heart disease. Circulation 1983;67:711-16.

3 Rashkind WJ. Interventional cardiac catheterization in congenital heart disease. Int $\mathcal{F}$ Cardiol 1985;7:1-11.

4 Lock JE, Rome JJ, Davis R, et al. Transcatheter closure of atrial septal defects. Circulation 1989;79:1091-9.

5 Rome JJ, Keane JF, Perry SB, et al. Double umbrella closure of atrial septal defects: initial clinical applications. Circulation 1990;82:751-8.

6 Sideris EB, Sideris SE, Tampoulos BD, et al. Transvenous atrial septal defect occlusion by the buttoned device. $A m \mathcal{F}$ Cardiol 1990;66:1524-6.
7 Sideris EB, Sideris SE, Fowlkes JP, et al. Transvenous atrial septal defect occlusion in piglets with a "buttoned" double disk device. Circulation 1990;81:312-18.

8 Hellenbrand WE, Fahey JT, Mc Gowan FX, et al. Transoesophageal echocardiographic guidance of transcatheter closure of atrial septal defect. Am f Cardiol 1990; 66:207-13.

9 Bridges ND, Hellenbrand W, Latson L, et al. Transcatheter closure of patent foramen ovale after presumed paradoxical embolism. Circulation 1992;86:1902-8

10 Lock JE, Cockerham JT, Keane JF, et al. Transcatheter umbrella closure of congenital heart defects. Circulation 1987;75:593-9.

11 Lloyd TR, Mendelsohn AM, Beekman RH, et al. Atrial septal defect occlusion with the buttoned device: early FDA trial results [abstract]. Circulation 1992;86:2518.

12 Das GS, Voss G, Jarvis G, et al. Experimental atrial septal defect closure with a new, transcatheter, self-centering device. Circulation 1993;88:1754-64.

13 Das GS, Shrivastava S, O'Laughlin, et al. Intermediate term follow up of patients after percutaeous closure of atrial septal defects with the Das angel wings device [abstract]. Circulation 1996;94:I-56.

14 Rao PS, Langhough R, Beekman RH, et al. Echocardiographic estimation of ballon-stretched diameter of secundum atrial septal defect for transcatheter occlusion. $\mathrm{Am}$ Heart 7 1992;124:172-5.

15 King TD, Thompson SL, Mills NL. Measurement of atrial septal defect during cardiac catheterisation: experimental and clinical trials. Am f Cardiol.

16 Sharafuddin MJ, Gu X, Titus JL, et al. Transvenous closure of secundum atrial septal defects: preliminary results with a new self-expanding nitinol prosthesis in a swine model. Circulation 1997;95:2162-8.

17 Boutin C, Musewe NN, Smallhorn JF, et al. Echocardiographic follow-up of atrial septal defect after catheter closure by double-umbrella device. Circulation 1993;88: $621-7$.

18 Carminati M, Hausdorf G, Tynan M, et al. Initial clinical experience of transcatheter closure of secundum atrial septal with a septal occlusion system. A multicenter European study [abstract]. Eur Heart $\mathcal{7}$ 1997; 18:136.

19 Zamora RRP, Lloyd TR, Beekmann RH, et al. Follow-up results of the multi-institutional phase I FDA supervised clinical trials of transcatheter occlusion of atrial septal
defects with buttoned device [abstract]. $7 \mathrm{Am}$ Coll Cardiol 1997;29:143.

20 Hausdorf G, Schneider M, Franzbach B, et al. Transcatheter closure of secundum atrial septal defects with the atrial septal defect occlusion system (ASDOS): initial experience in children. Heart 1996;75:83-8.

21 Sievert H, Dirks J, Rux S, et al. ASD and PFO closure in adults with the second generation ASDOS device [abstract]. F Am Coll Cardiol 1997;29:143.

22 Sharafudin MJ, Gu X, Titus JL, et al. Secundum-ASD closure with a new self-expanding prosthesis in swine [abstract]. Circulation 1996;94:I-57.

23 Chan KY, Godman MJ, Wilson N, et al. Transcatheter closure of atrial septal defect with a new nitinol double disk device (Amplatzer septal occluder). United Kingdom experience. Eur Hearts F 1997;18:135

24 Horvath KA, Burke RP, Collins JJ Jr, et al. Surgical treatment of adult atrial septal defect: Early and long-term results. 7 Am Coll Cardiol 1992;20:1156-9.

25 Pastorek JS, Allen HD, Davis JT. Current outcomes of surgical closure of secundum atrial septal defect. Am $\mathcal{F}$ Cardiol 1994;74:75-7.

26 Meijboom F, Hess J, Szatmari A, et al. Long-term follow-up (9 to 20 years) after surgical closure of atrial septal defect at a young age. Am f Cardiol 1993;72:1431-4.

27 Konstantinides S, Geibel A, Olschewski M, et al. A comparison of surgical and medical therapy for atrial septal defect in adults. N Engl f Med 1995;333:469-73.

28 Ward C. Secundum atrial septal defect: routine surgical treatment is not of proven benefit. Br Heart f 1994;71:21923

29 Galal MO, Wobst A, Halees Z, et al. Peri-operative complications following surgical closure of atrial septal defect type II in 232 patients-a baseline study. Eur Heart $\mathcal{f}$ 1994;15:1381-4.

30 Young D. Later results of closure of secundum atrial septal defects in children. Am 7 Cardiol 1973;31:14-22.

31 Sellers RD, Ferlic RM, Sterns LP, et al. Secundum type atrial septal defects: early and late results of surgical repair using extracorporal circulation in 275 patients. Surgery 1966;59:155-64. 